\title{
Nationwide Impact and Vehicle to Grid Application of Electric Vehicles Mobility using an Activity Based Model
}

\author{
Roberto Álvaro, Jairo González, Jesús Fraile-Ardanuy
}

\author{
Luk Knapen, Davy Janssens
}

\begin{abstract}
This paper describes the impact of electric mobility on the transmission grid in Flanders region (Belgium), using a micro-simulation activity based models. These models are used to provide temporal and spatial estimation of energy and power demanded by electric vehicles (EVs) in different mobility zones. The increment in the load demand due to electric mobility is added to the background load demand in these mobility areas and the effects over the transmission substations are analyzed. From this information, the total storage capacity per zone is evaluated and some strategies for EV aggregator are proposed, allowing the aggregator to fulfill bids on the electricity markets.
\end{abstract}

\section{INTRODUCTION}

Electric mobility is becoming an option for reducing greenhouse gas emissions of road transport and decreasing the external dependence on fossil fuels. However, this new kind of mobility will introduce additional loads to the power system.

Investments in electricity networks are very expensive and costly in time; therefore it is important to anticipate the impact of a massive deployment of electric vehicles in the electric grid. At the same time, electric companies plan their electricity networks assuming that these consumptions in different nodes of the electric grid are varying continuously in time but are static in space. With the introduction of electric vehicles $(\mathrm{EVs})$ and their inherent mobility, there will also be a remarkable spatial variation in the electric loads that could increase the total stress on the power grid. This stress can also cause changes in the behavior of electric vehicle drivers, because they may not be able to recharge at the desired locations and at the desired times due to charging restrictions.

At the same time, EVs are parked, on average, more than $80 \%$ of the day [1], so they can be used as distributed storage systems using bidirectional chargers, selling capacity and energy on the electric market and providing ancillary services to the grid. This concept is commonly called as Vehicle to Grid (V2G) [2-4].
Due the small capacity of a single $\mathrm{EV}$, an aggregator agent is required between the system operator ( $\mathrm{SO}$ ) and the thousands of EVs owners. The aggregator will participate in the electricity market with supply and demand energy bids.

The main objectives of this paper are:

- Firstly, to analyze the effect of a massive EVs deployment in the Flemish electric grid, having accurate information about the spatial and temporal electric load demand due to the electric mobility.

- Secondly, to estimate the distributed storage capacity available in the Flemish grid that can be handled by an EV aggregator. This aggregator must develop a good estimation of several EVs uncertainties like total EV electrical energy consumption, total battery state of charge and number of EV plugged in each hour and in each zone using the mobility information, because it must associate the market bid to a transmission network node [5].

\section{ACTIVITY BASED MODEL}

The temporal and spatial behavior of EVs is modeled using an activity-based (AB) micro-simulation model, called Feathers, developed in [6] by the Transportation Research Institute (IMOB) in Hasselt University.

This model allows predicting daily schedules for people over a nationwide region under study. In this case, a synthetic population of different agent is built, where every agent simulates an inhabitant of this region. The behavioral characteristic for each synthetic inhabitant is based on real travel behavior OVG surveys, generating a very precise microsimulation model of activity-travel demand. Check references [6] and [7] for a more detailed description of this model.

The $\mathrm{AB}$ mobility model divides the Flanders region (6 million inhabitants on $13000 \mathrm{~km}^{2}$ ) in 2368 different zones with 
an average area of $5.5 \mathrm{~km}^{2}$ per zone and its output is a complete schedule list for the whole population. Each schedule is composed by an activity - trip associated with a specific member of this population. This schedule contains information related to the kind of activity (work, shopping, bring-get, etc., although in this study only work or non-work activities were distinguished), trip information (departure time, trip duration, origin and destination zones, traveled distance, etc.) and transportation mode (by foot, by train, by car, etc.).

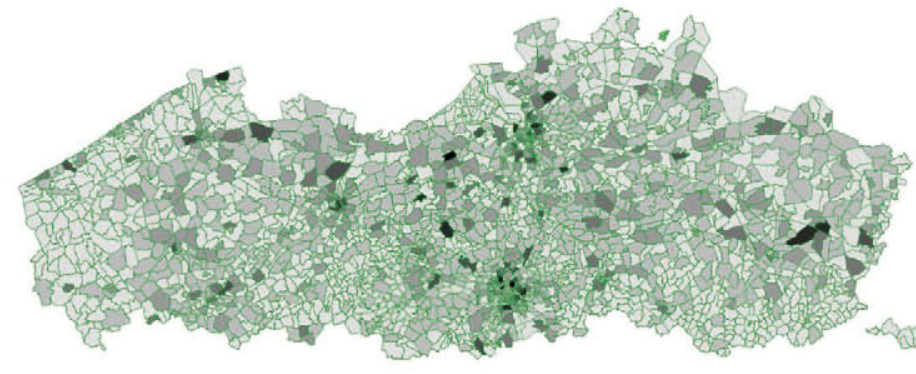

Fig. 1: Peak power demand due to EV only, during the low tariff hours [1].

To estimate the electricity energy demand and the peak power demand as a function of time and location due to EVs deployment, some assumptions related to the total battery capacity of these vehicles, nominal energy consumption and nominal power of chargers were done in [7].

From these assumptions, different charging scenarios (i.e. start charging as soon as the car arrives home, star charging as soon as the low tariff period starts, etc.) and different market penetration scenarios for EVs were analyzed and the peak power demand and the energy demand were evaluated for each scenario.

Fig. 1 shows the peak power demand during low tariff hours for each Flemish mobility region. Darker areas on the map correspond to higher electric energy consumption due to EVs charging.

\section{DISAGGREGATION OF THE EXISTING BACKGROUND ELECTRICITY DEMAND AND THE FLEMISH TRANSMISSION ELECTRIC GRID}

Although the main impact of EVs on the power system will be more important on the electric distribution network, the granularity of the activity-based model (with 2368 mobility zones with an average surface of $5-6 \mathrm{~km} 2$ ) prevents to analyze this impact in such detail. Therefore, the analysis will be specifically focused on the Flemish transmission network.

The first step to evaluate possible problems on this electric network is to add the electricity demand due to EVs for each zone (from [7] and shown in Fig. 1) to the already existing background electricity demand for each mobility zone. Unfortunately, only countrywide aggregated time-depending electricity consumption data is easily available and therefore, the total countrywide existing background electricity demand must be spatially disaggregated for each mobility zone firstly.

\section{A. Disaggregation of existing background electricity demand}

For each mobility zone, the total time-depending electricity energy demand will be divided in three different components:

- EV load demand per zone, obtained from the ABmodel [7].

- Domestic load demand per zone.

- Additional electric load demand per zone.

1) Domestic load per zone evaluation

To estimate the time depend domestic load demand per mobility zone, the annual synthetic average domestic load per household in Flanders, with one-hour resolution was obtained from the Flemish Regulator of Electricity Market [8].

The population available in each mobility zone was obtained from the AB-model and statistics about population. Demographic data from the Eurostat website [9] were used to evaluate the total number of households per mobility zone. The number of households per zone was determined by evaluating the quotient between the population per zone and the average number of people per house in Flanders [10].

Multiplying the number of households by the annual synthetic average domestic load per house [8], an initial total domestic load per mobility zone was obtained. In this case, all mobility areas had the same consumption profile but multiplied by a scaling factor proportional to the population per area.

In order to avoid that all mobility areas have the same, but weighted, consumption profile, some variability was added to each profile based on real measurements extracted from 2500 smart meters provided by a Spanish distributor operator. Assuming the annual synthetic average domestic load per household in Flanders as an average value, new domestic demand profiles were generated for each household in each mobility area based on the variance evaluated in this Spanish case. The sum of all these domestic consumptions per household was the total domestic demand per mobility zone.

\section{2) Additional Domestic load per zone evaluation}

Since no information about the actual industrial electrical consumption per zone was available, it was assumed that all electric demand in Belgium due to this type of loads was consumed almost homogeneously along the country with a higher ratio in areas located in Flanders. This takes into account that, according to statistics about industrialization of main regions of Belgium, extracted from EUROSTAT website [9], the Flemish region is more industrialized than the rest of the country.

The total electric demand for Flanders was evaluated by multiplying the total electric demand for the whole country, available on TSO Elia website [10], for a scaling factor proportional to the Flanders population. Rest of electric consumption in Flanders region, was evaluated by subtracting the total electric demand in Flanders and the total electric domestic demand for all mobility zones in Flanders. The electric demand profile of the industrial electric consumption for each mobility zone was obtained multiplying the rest of the electric consumption in Flanders region by a zone ratio defined 
as the ratio between the population per zone and the total Flemish population.

\section{B. Flemish transmission electric grid}

The transmission electric grid in Flanders consists of 179 transmission substations with several voltage levels from 380 $\mathrm{kV}$ to $70 \mathrm{kV}$. The electric capacity of these substations is between 40 MVA to 800 MVAs [12]. These substations have a nominal power capacity that should not be exceeded under any load scenario, so during the simulation and analysis, this condition is checked for each substation.

Since there are more defined mobility zones than transmission substations, several mobility areas are fed by the same substation and an algorithm to assign mobility zones to the nearest substations based on the closest distance between the mobility area centroid and the substation location was developed (see Fig. 2).

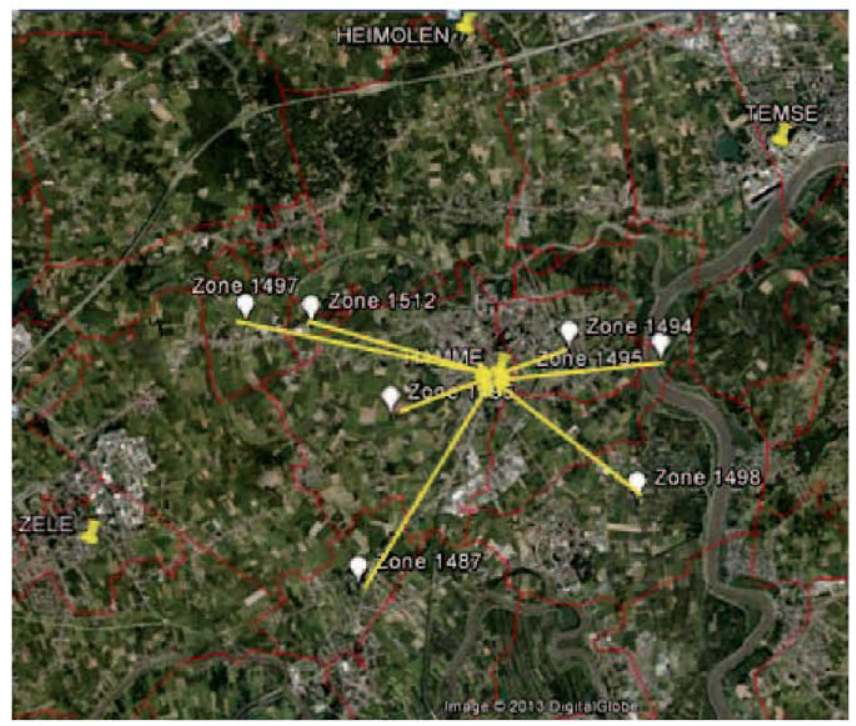

Fig. 2. Assignment of mobility zones to the nearest transmission substation

\section{DISTRIBUTED STORAGE CAPACITY}

Whether in the present electricity markets or in future scenarios, by developing optimal bid strategies and satisfy the operation system rules [5] the EV aggregator must forecast for each instant time the stored energy that is plugged into grid, the capacity to storage energy plugged in and where vehicles are located.

From [2] it is known that suitable services to cover by EV aggregator are regulation and storage. Therefore, in order to analyze the net revenue if ancillary services are provided and participate in the electricity market placing correct bids, EV aggregator must forecast some key information related to the EVs that are pooled under its control.

The main required information is: the total battery capacity of each EV, the minimum SoC needed at the departure time of each $E V$, the total available storage capacity per hour (which will depend on the distance traveled by the car) and the connection capacity.
From the electric mobility model proposed in [6], this required information can be estimated for each mobility zone, and therefore by network node, allowing the EV aggregator to program its market bids and to develop business models promoting EVs owners to maintain the EVs plugged in during the parked times.

Some assumptions have been done to obtain the storage capacity by zone available to offer upward and downward regulation:

-Feathers model simulates mobility by day and not some days in a row. Due to that, it has been supposed all vehicles begin the day completely charged.

-Only vehicles with assigned activities and trips that can be covered without recharging during the day are selected to offer storage capacity for the aggregator, so the regulation down capacity is underestimated in this case.

-To evaluate the storage capacity to offer regulation up, it has been taken into account the minimum SoC that each vehicle needs to cover all its expected trips.

-Feathers model does not consider vehicles that are parked during all the day, so it is not possible to estimate the total number of them. This set of vehicles could offer regulation points fixed (e.g., at home, at work, etc.) to EV aggregator.

-The possibility that all storage capacity could be available will depend on several aspects as power infrastructure that works with this new load level, availability of chargers, nominal power of chargers and location of chargers. In this way, that is a question of investment, since the technology is currently available. Therefore, it is assumed that each vehicle is plugged into grid through a bidirectional charger that can deliver all the stored energy in one hour. In other words, the available capacity coincides with the capacity to regulation.

-Penetration rate used in this study is always the relation between the number of battery electric vehicles and the total fleet. It is important to highlight that depending on the type of the electric vehicle, the offered services that can be covered are not the same. For example, plug-in hybrid cars have a very small battery capacity so only regulation up, starting the ICE engine, can be offered in this case [2], [5].

From the mobility model, it is obtained the number of electric vehicles, their $\mathrm{SoC}$ and the zones where they are located in each time of the day. Processing this data, the vehicles suitable to cover regulation under assumptions enumerated above are used to estimate the capacity to storage energy (regulation down) and capacity to supply energy (regulation up) aggregated by zone along the day.

In the figure 3 it is illustrated the different parameters that should know EV the aggregator by zone and time. 


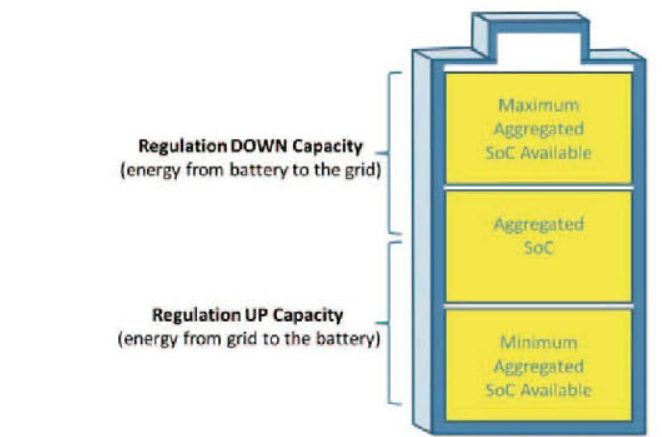

Fig 3. Parameters necessary to cover regulation by EV aggregator

\section{RESULTS}

\section{A. Analysis of the EVs mobility on the Flemish transmission network}

Several mobility and charging scenarios are simulated in this work, analyzing the effect of the total electric demand on the Flemish electric grid.

The analysis has been focused in the two following cases: under similar charging scenarios with different EV market share and under identical EV market share and different charging scenarios.

Two scenarios are used:

-Scenario 1. People start charging as soon as possible during the low tariff period.

-Scenario 2. People start charging uniformly distributed in time, adopting a decentralized smart grid strategy to avoid problems in the electric grid.

Vehicle electrification rate simulated is divided in two different cases: case $1,5 \%$ of EVs rate penetration, and case 2, $10 \%$ of $\mathrm{EV}$ rate penetration.

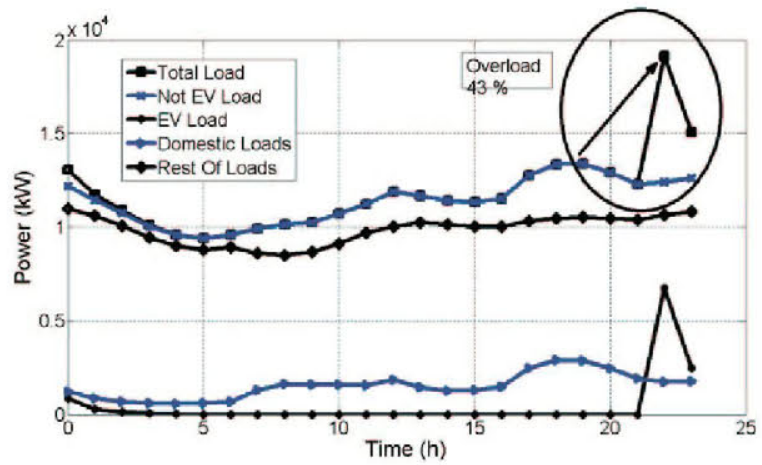

Fig. 4. Charging Scenario 1 in case 2

The main results to highlight are the following:

-The mobility zone with the highest electric demand does not have to be supplied by the most loaded substation neither by the substation which whose load is the most increased by the penetration of EVs.

-At transmission grid level, no problems have been observed in the substations, taking into account the operational constraints.
-It has been observed an increment in the average load in the transmission substation during some hours. In some critical scenarios, this increment can reach up to $25-30 \%$ in some substations.

-The charging scenario has a great incidence on the power demand profile. For example, in Fig. 4 and 5 it is shown the total power demand profile for the different charging scenarios under same penetration rate for the same transmission substation. Under an uncontrolled charging scenario (scenario 1), shown in Fig. 4, a high peak at the start of the low tariff period is produced in this profile. With a controlled charging scenario (scenario 2), this energy is share among different hours and the high peak is avoided.

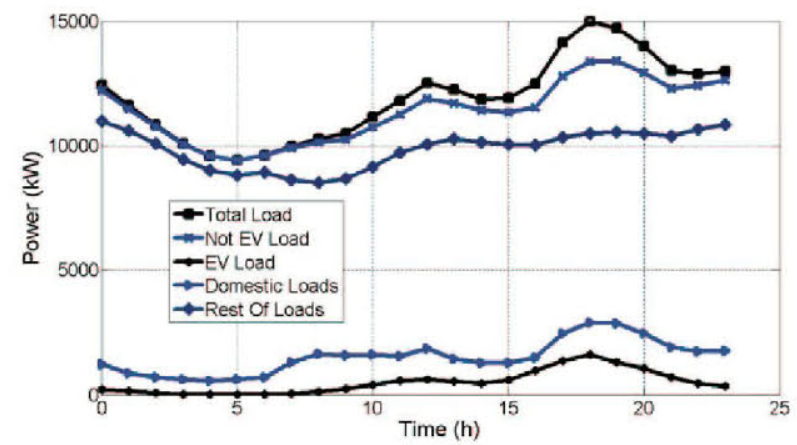

Fig. 5. Charging Scenario 2 in Case 2

\section{B. Evaluation of the distributed storage capacity}

Under assumptions commented in section IV, several results have been obtained from the simulations. As it is shown in Fig. 6, the capacity to regulation up at the beginning of the day is high and decreases along the day because the batteries are completely full in the early morning. Meanwhile regulation down profile is the opposite because EVs are used during the day and their batteries are almost empty in the late evening.

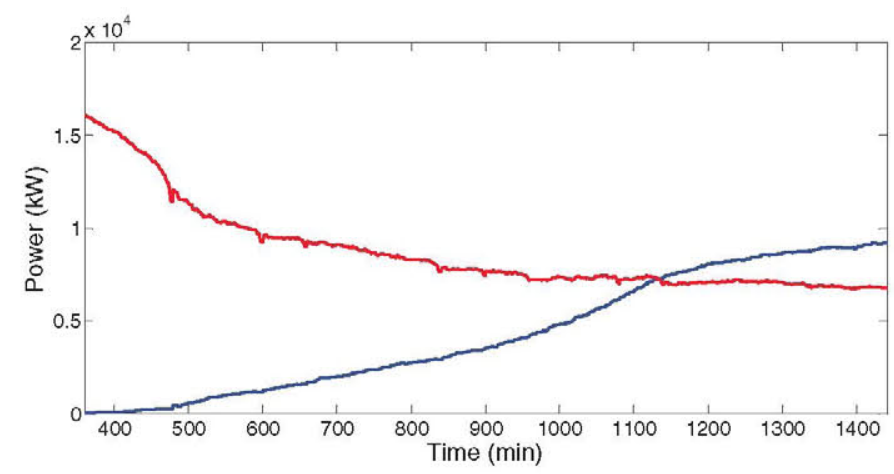

Fig. 6. Regulation Up (red) and Down (blue) Capacity in Zone 2386

It can be also observed that with the highest penetration rate simulated $(100 \%)$, the regulation capacity is very high. For example, between $16 \mathrm{MW}$ and $7 \mathrm{MW}$ in the case of zone 2386 , as it is showed in Fig. 6. If these values are compared with the nominal power of the transmission substation which is feeding this particular area, $40 \mathrm{MW}$, it is possible to assess the potential of the distributed storage that EVs could have. 
On the other hand, in Fig. 7 and 8 it is showed the aggregated regulation up and down capacity of whole Flanders. As it can be seen in these figures, the values of power that can be managed to offer regulation services reaches magnitudes of $\mathrm{GW}$, which are much higher than the mean maximum imbalance assumed by TSO Belgian (Elia) for the correct operation of the network.

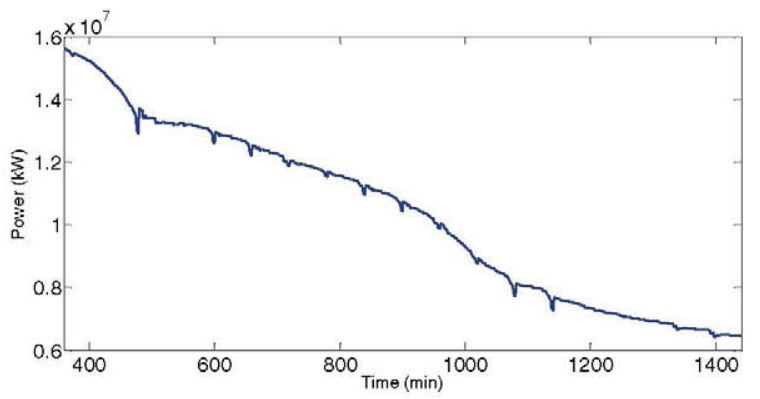

Fig. 7. Total Regulation Up Capacity. Max=16 GW; Min=6.5GW

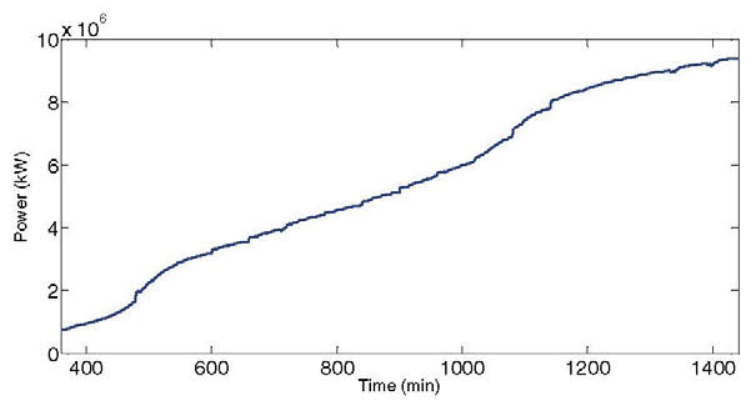

Fig. 8. Total Regulation Down Capacity. $\mathrm{Max}=9.5 \mathrm{GW}$; Min $=0.8 \mathrm{GW}$

\section{CONCLUSIONS AND FUTURE WORK}

The impact on the substations in the electric transmission system has been studied in a large number of charging scenarios. Due to limitations in the full access to the electric grid information, some assumptions have been made. Under these conditions the results are not worrisome, in the way that problems related to the collapse in any node of the transmission grid have not been found.

In any case, it is important to comment that the increment in the level of the substation load reaches in some of them up to $35 \%$ in some analyzed critical scenarios, raising the average load in some cases from $50 \%$ to $65 \%$.

From the TSO point of view, this increment in most of the substations could generate an increment of the global losses of the system and possible reinforcements in the grid could be necessary in some particular points to better manage the voltage level if no other strategies are considered. That is an assumption that would need to be corroborated with a deeper study having higher access to the electric grid data.

On the other hand, for the EV aggregator's point of view, it is essential to know with accuracy how much and where it is located the distributed storage capacity. Regarding the power infrastructure, in this study is showed that for the EV aggregator is necessary to analyze if all energy storage in certain zones could be delivered at the same time, because the aggregated capacity of the mobility zones assigned to one particular substation could reach high levels of power flow compared with the substation nominal power.

In addition, in this study it is demonstrated that the potential to cover regulation in the Belgian electricity market by EVs is very high, being estimated that it would be only necessary between $5 \%$ and $15 \%$ of vehicle Flemish electrification rate with permanent access to a charger every time that they are parked.

It is important to know the exact location of the aggregated EVs for other future electricity related markets, for example to sell energy to DSO, or in scenarios with high penetration of distributed generation and renewable energies

\section{ACKNOWLEDGMENT}

The research leading to these results has received funding from the European Union Seventh Framework Programme (FP7/2007-2013) under grant agreement n 270833.

\section{REFERENCES}

[1] J. Van Roy, N. Leemput, S. De Breucker, F. Geth, P. Tant, J. Driesen, "An Availability Analysis and Energy Consumption Model for a Flemish Fleet of Electric Vehicles", European Electric Vehicle Congress, 26-28 October, 2011, Brussels.

[2] W. Kempton and J. Tomic, "Vehicle-to-grid power fundamentals: Calculating capacity and net revenue", Journal of Power Sources, vol. 144, no 1, pp. 268-279, Jun. 2005.

[3] W. Kempton and J. Tomic, "Vehicle-to-grid power implementation: From stabilizing the grid to supporting large-scale renewable energy", Journal of Power Sources, vol. 144, no 1, pp. 280-294, Jun. 2005.

[4] W. Kempton, V. Udo, K. Huber, K. Komara, S. Letendre, S. Baker, D. Brunner and N. Pearre, "A Test of Vehicle-to-Grid (V2G) for Energy Storage and Frequency Regulation in the PJM System", University of Delaware, Tech. Report, 2008.

[5] R. J. Bessa and M. A. Matos. "The Role of an Aggregator Agent for EV in the Electricity Market", $7^{\text {th }}$ Mediterranean Conference and Exhibition on Power Generation, Transmission, Distribution and Energy Conversion, MED10, 7-10 November, 2010. Cyprus.

[6] T. Bellemans, B. Kochan, , D. Janssens, G. Wets, T. Arentze and H. Timmermans "Implementation Framework and Development Trajectory of FEATHERS Activity-Based Simulation Platform" Transportation Research Record: Jorunal of the Transportation Research Board, Vol 2165, 17 2010, pp. 111-119.

[7] L. Knapen, B. Kochan, T. Bellemans, D. Janssens, G. Wets, “Activity based models for countrywide electric vehicle power demand calculation" IEEE First International Workshop on Smart Grid Modeling and Simulation (SGMS), Brussels (Belgium), 17 Oct 2011, pp. 13-18, 2011.

[8] http://www.vreg.be/verbruiksprofielen-0

[9] http://epp.eurostat.ec.europa.eu

[10] http://www.elia.be/en/grid-data/data-download

[11] J. Fraile-Ardanuy, D. Ramírez, S. Martínez, R. Álvaro, J. González, L. Knapen and D. Janssens, "Impacts of Electric Mobility and Electric Grid", Data on Science and Simulation in Transport Research, DSSTR 2014 (to be published)

Pieter Schavemaker and Lou van der Sluis, Electric Power System Essentials, John Wiley \& Sons, 2008. 